\section{Class II corrections made easy}

Despite being some of the more common orthodontic challenges, Class II corrections can be challenging to treat.

With the 3M Forsus Class II Correction System, you can rely on predictable, easy treatment for these cases.

Able to be fitted in a single

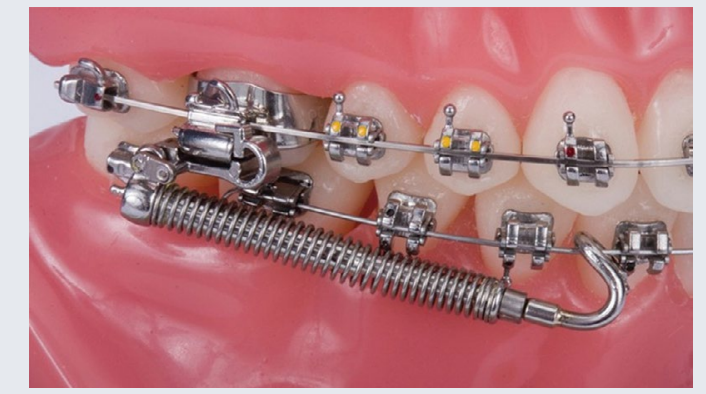
appointment without the need for any additional lab work, the 3M Forsus Class II Correction System has been specifically designed for optimal patient comfort. The open spring design facilitates oral health maintenance during treatment, while independent left and right components mean that you can install only one side if necessary, for a more tailored approach.

To find out more, contact $3 \mathrm{M}$ today.

For more information, call 08458734066 or visit https://www.3m.co.uk/3M/en_GB/p/d/ b5005051009/.

$3 \mathrm{M}$ and Forsus are trademarks of the $3 \mathrm{M}$ Company.

\section{Specialist payroll providers}

Prepare for the unexpected with Wagemate!

Sometimes events are out of our control, but as specialist payroll providers, Wagemate can cope with anything. Wagemate's award-winning disaster recovery system is tried and tested for peace of mind.

When you outsource to Wagemate, no matter what life throws your way, payroll will run smoothly and your key data will be safe and secure.

Wagemate manage everything, from BACS to pensions and benefits. They have many happy dental clients who have grown with them over the years, because they offer flexibility as part of their quality service.

Life is full of surprises - call Wagemate today.

To better manage your payroll, contact payroll specialists Wagemate today, tel: 03330 102102 or email info@wagemate.com.

\section{Endodontics without fear}

For fearful or anxious patients, it can help to give a breakdown of each treatment step, the tools and processes you will use.

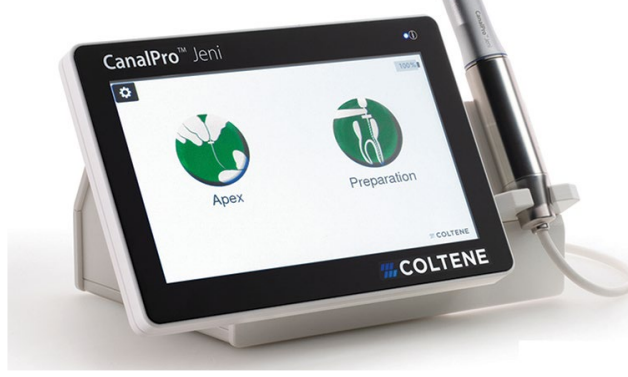

This is particularly important with treatments like root canal therapy.

COLTENE has everything a specialist needs to deliver comfortable, efficient and successful endodontics. The patient will be healed, with a route out of pain.

The COLTENE endo range includes:

- The CanalPro Jeni, a combined endometer/apex locator for autonomous digital navigation in the root canal

- HyFlex EDM files, including the REMOVER file for mechanical retreatments

- Materials for confident obturation and sealing.

Create a reputation for empathetic endodontic that endures. Talk with COLTENE for more.

For more on COLTENE, visit www.coltene. com, email info.uk@coltene.com or call 0800 2545115 .

\title{
Save money and improve your work-life balance with the cloud
}

The recent State of Dentistry report conducted by Dentally (https://info. dentally.co/smile-2021-0) found that over $75 \%$ of dentists believe that technology can help them to establish greater flexibility in their work-life balance. Dentally aims to achieve that. With their cloud-enabled practice management, you can be sure that versatility is simple and easy to implement into your practice, all whilst saving money.

As your practice slowly returns to some form of normality - footfall increases, chairs fill and diaries become busy once more, you will, of course, be wishing to save money where possible. With so many missed appointments and patients in need of dental care, it is more important than ever for you as a practice to provide exceptional care to all patients, whilst reducing costs within your business and ensuring you are optimising all of your practice workflows.

It is undeniable that the effects of COVID-

19 have presented those in the dental industry with some of the most challenging times of their careers thus far. Dentally want to ensure their customers know that ease of use, saving money and saving time are at the heart of all they do. With their cloud-enabled practice management software, Dentally make running your practice simple, easy and cost-effective.

From automated workflows, customisable permission settings, detailed reports and

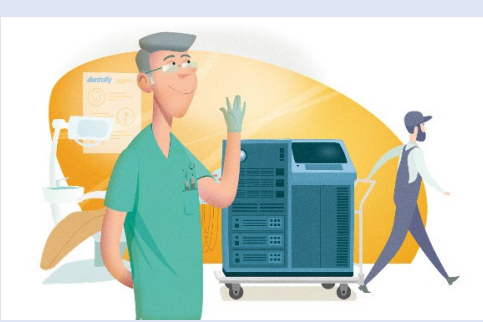

remote working - Dentally's flexible toolkit means you can run the practice you want with just a little help from the cloud.

Talk to the Dentally team today at hello@dentally.co to find out how the UK's leading cloud-enabled practice management software can change your practice for the better.

For more information visit: https:// dentally.co/ or call 02038565610 . 